\section{Fluorescence nanoscopy by ground-state depletion and single-molecule return}

\author{
Jonas Fölling ${ }^{1,2}$, Mariano Bossi ${ }^{1,2}$, Hannes Bock ${ }^{1}$, \\ Rebecca Medda ${ }^{1}$, Christian A Wurm ${ }^{1}$, Birka Hein ${ }^{1}$, \\ Stefan Jakobs ${ }^{1}$, Christian Eggeling ${ }^{1} \&$ Stefan W Hell ${ }^{1}$
}

\begin{abstract}
We introduce far-field fluorescence nanoscopy with ordinary fluorophores based on switching the majority of them to a metastable dark state, such as the triplet, and calculating the position of those left or those that spontaneously returned to the ground state. Continuous widefield illumination by a single laser and a continuously operating camera yielded dual-color images of rhodamine- and fluorescent protein-labeled (living) samples, proving a simple yet powerful super-resolution approach.
\end{abstract}

For many decades it was assumed that the resolution of any far-field optical microscope is limited to about half the wavelength of light. This perception has changed after the discovery that basic fluorophore transitions can be used for neutralizing the limiting role of diffraction ${ }^{1,2}$. More specifically, transitions that switch the fluorescence on or off enabled the sequential recording of objects that are much closer than the diffraction limit. Hence, not surprisingly, all far-field fluorescence nanoscopy modalities used so far rely on a time-sequential readout using a variant of fluorescence switching. In stimulated emission depletion (STED) microscopy ${ }^{1}$, the fluorescence ability of the dye is switched using a de-excitation beam. In ground state depletion (GSD) microscopy ${ }^{2,3}$, the fluorophores are switched to the dark triplet state. In contrast to STED, saturated patterned excitation microscopy (SPEM or SSIM) ${ }^{4,5}$ switches the fluorescence to its maximum. All of these strategies have been extended to switching photoactivatable fluorescent proteins and photoswitchable (photochromic) organic fluorophores ${ }^{6}$. They all switch the fluorescence using a light distribution featuring either one or many intensity zeros that are translated in space, defining the coordinates at which fluorescence is switched on or off at a given point in time ${ }^{7}$.

This is different in photoactivation localization (PALM $)^{8,9}$ or stochastic optical reconstruction microscopy (STORM) ${ }^{10}$ where the fluorescence ability of the marker is switched stochastically in space, molecule by molecule. The fluorescence diffraction pattern of sparsely and randomly switched on (activated) molecules is recorded on a camera, allowing the calculation of their position with accuracy $\Delta / \sqrt{ } m$, with $\Delta$ denoting the width of the diffraction maximum and $m$ the number of detected photons ${ }^{11}$. Clearly, for this concept to work, the molecules must cycle from a dark to a bright state, yielding $m$ detectable photons, and then back to a dark state. Therefore, PALM and STORM use 'photoactivatable' proteins or organic compounds where the fluorescence ability of the molecule is elicited by the absorption of a photon. The energy provided by this 'activation' photon switches the fluorophore on, for example, by altering a chemical bond or an isomerization state. Examples of such compounds are the photoactivatable proteins $\mathrm{EosFP}^{8}$ and PA-GFP', the 'caged' rhodamines ${ }^{12}$ and the photoisomerizable cyanine dyes Cy3 and Cy5, which have been used both as activator-emitter pairs ${ }^{10}$ as well as single photoactivatable labels ${ }^{13,14}$

Although they have provided stunning images, these photoactivatable compounds have limitations in terms of biocompatibility, labeling and switching performance. Additionally, they may call for dedicated activation lasers ${ }^{8-10,12,14}$. Last but not least, the apparent need for 'activatable' compounds narrows the scope of these techniques. Here we show that far-field fluorescence nanoscopy by stochastic single-molecule switching can be performed without photoactivation, using basic transitions of standard markers: switching the fluorophores to their triplet state $T_{1}$ or another metastable dark state while recording those that are still left or have returned to the ground state $S_{0}$. Operating with ordinary fluorophores and rendering photoactivation either optional or obsolete, ground state depletion microscopy followed by individual molecule return (GSDIM) considerably expands the conceptual range and the applicability of far-field optical nanoscopy.

Having a lifetime $(\tau)$ of $10^{-3}$ to $100 \mathrm{~ms}$ and being common in virtually all fluorophores, the lowest triplet state $T_{1}$ is the primary candidate for switching molecules ${ }^{2}$. In addition, $T_{1}$ serves as a gateway to other dark states $D$ with similar or even longer $\tau$ of 1 to $10^{4} \mathrm{~ms}^{15}$ (Fig. 1a). After the period $\tau$, the molecules return to $S_{0}$ where they can be repeatedly excited to the fluorescent state $S_{1}$, yielding the burst of $m$ detectable photons required for computing their position. The same repeated excitation also switches the fluorophore off, because from $S_{1}$ the molecule crosses to $T_{1}$ with a typical probability $\Phi_{\text {isc }} \leq$ $0.1 \%$. If $\tau$ is $\sim 10^{7}$ times longer than the fluorescence lifetime $\left(\tau_{\mathrm{fl}}\right)$ of $\sim 3$ ns of $S_{1}$, a continuous wave illumination intensity $I>I_{\mathrm{s}} \equiv h v /\left(\Phi_{\text {isc }}\right.$ $\sigma \tau) \approx 1 \mathrm{~kW} / \mathrm{cm}^{2}$ minimizes the fraction of $S_{0}$ molecules to $\varepsilon \approx \tau_{\mathrm{fl}} / \Phi_{\text {isc }}$ $\tau<<10 \%$; $h v$ is the energy of the excitation photon and $\sigma$ the photon absorption cross-section of the molecule in $S_{0}$. Note that the minimal fraction $\varepsilon$ of remaining $S_{0}$ molecules does not depend on $I$.

Owing to the presence of a larger dark-state population, the signal from a bulk of rhodamine dyes, specifically Rhodamine 6G (Rh6G) and Atto532 embedded in poly(vinyl-alcohol) (PVA), decreased with increasing intensity of the 532-nm excitation beam used (Fig. 1b). Notably,the majority of the dyes recovered within several tens of milliseconds (Fig. 1c). Addition of the triplet quencher

${ }^{1}$ Max Planck Institute for Biophysical Chemistry, Department of NanoBiophotonics, Am Fassberg 11, 37077 Göttingen, Germany. ${ }^{2}$ These authors contributed equally to this work. Correspondence should be addressed to S.W.H. (shell@gwdg.de) or C.E. (ceggeli@gwdg.de).

RECEIVED 24 JULY; ACCEPTED 2 SEPTEMBER; PUBLISHED ONLINE 15 SEPTEMBER 2008; DOI:10.1038/NMETH.1257 
a

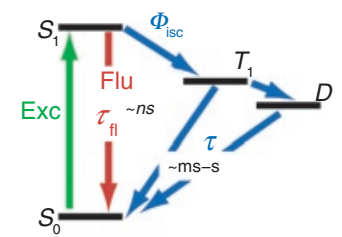

C

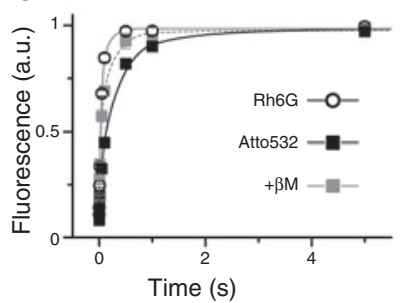

b

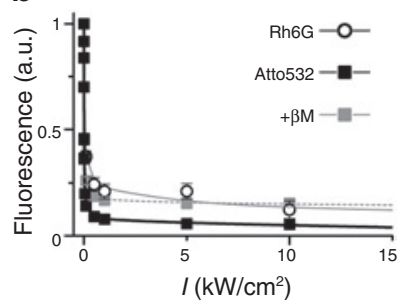

d

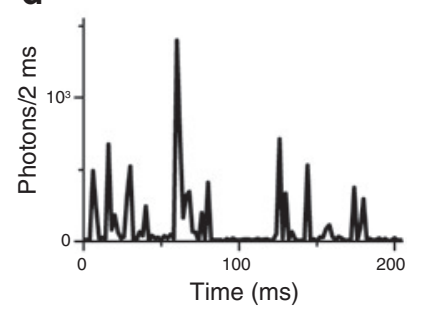

Figure 1 | Switching a regular fluorophore using its dark states. (a) Repetitive excitation (Exc) of the singlet ground state $S_{0}$ to the first excited singlet state $S_{1}$ elicits fluorescence (Flu) emission and switches a molecule to the triplet state $T_{1}$ or other dark states $D$ with long lifetime $(\tau)$. (b) GSD of Rh6G, Att0532 and Att0532 plus a triplet quencher ( $\beta$-mercaptoethanol; $\beta$ M) for increasing intensity, $I$. (c) Recovery of the active fluorophores after maximal dark state shelving (see Supplementary Table 1). (d) Fluorescence time trace of a single Atto532 molecule in PVA given as the number of detected photons per 2-ms time bin $\left(I=115 \mathrm{~kW} / \mathrm{cm}^{2}\right)$.

$\beta$-mercaptoethanol reduced $\tau$ and hence increased $\varepsilon$. This experiment demonstrated the critical role of $T_{1}$ in the switching mechanism. On a single-molecule level the transient population of $T_{1}$ or $D$ induced a fast stochastic on-off switching; the average on-times were a few milliseconds and the off-times were somewhat larger (Fig. 1d). The time spent in the fluorescent (on) singlet system was $<10 \%$ of the total recording time. Yet the number of photons detected per $2 \mathrm{~ms}$ on time amounted to $m>500$ because once they were back in the singlet system, the fluorophores emitted brightly, with the distribution of $m$ peaking at 1,000 photons.

Dual-color GSDIM images of microtubule filaments and peroxysomes of mammalian cells embedded in PVA, immunolabeled with the rhodamine derivatives Atto532 (emission maximum 550 nm) and Atto565 (emission maximum $\sim 590 \mathrm{~nm}$ ) showed details absent in conventional images (Fig. 2a,b). We recorded the images using a 532-nm continuous-wave laser for both excitation and depletion, and a continuously running camera. The resolution of the GSDIM images was $<30 \mathrm{~nm}$ (Supplementary Methods online).

The $40 \mathrm{~nm}$ disparity between the emission peaks of both dyes was sufficient to distinguish them with a confidence $>90 \%$ when comparing the signal of a single isolated emitter detected in a short-pass $(<575 \mathrm{~nm})$ channel with that in a long-pass $(>590 \mathrm{~nm})$ channel $^{16}$. The conventional bulk recording requires mathematical unmixing.

The fact that the photon burst $m$ is provided by the singlet system of a regular dye explains why $m$ may easily exceed 500 photons. As $\mathcal{E} \approx \tau_{\mathrm{fl}} / \Phi_{\mathrm{isc}} \tau$, large values of $\tau$ and $\Phi_{\mathrm{isc}}$ of a fluorophore improve the switching, but a large $\Phi_{\text {isc }}$ also reduces $m$, which is approximated by $\eta_{\mathrm{det}} \Phi_{\mathrm{fl}} / \Phi_{\text {isc }}$, and hence independent of $I$. $\left(\eta_{\text {det }}\right.$ and $\Phi_{f l}$ denote the detection efficiency of the instrument and the fluorescence quantum yield, respectively.) Therefore, we used dyes with $\Phi_{i s c}<0.1 \%$

and a large $\tau$. The latter is provided by the PVA environment, which reduces the mobility of triplet-quenching oxygen and yields additional dark states ${ }^{15}$. Many fluorophores mounted in PVA, including Alexa488, Texas Red, FITC, Rhodamine1 10 and Oregon Green yielded $\varepsilon \leq 10 \%$ and $\tau$ greater than milliseconds; they all are suitable for GSDIM (Supplementary Table 1 online). $m$ is likely larger than in genuine photo-switchable fluorophores because the probability of the latter to go to an off state is usually higher. GSDIM benefits from the fact that standard fluorophores are optimized for large fluorescence quantum yields.

Next we immersed our samples in Tris buffer containing glucose oxidase as oxygen scavenger, which, by increasing $\tau$ from about microseconds to $\sim 10-100 \mathrm{~ms}$, enabled $\varepsilon<10 \%$ (Supplementary Table 1). The GSDIM images of microtubules immunostained with Rh6G displayed a superior resolution (Fig. 2c). GSDIM was also possible in cellular media such as HEPES-buffered Dulbecco's modified Eagle's medium with an oxygen-scavenging system. Clusters of integrin- $\beta-3$ of human glioma cells labeled with Atto532 were far better resolved by GSDIM than by conventional recording (Fig. 2d,e).

Fluorescent proteins can also be efficiently transferred to a metastable dark state such as the triplet or different protonation states ${ }^{17}$. All fluorescent proteins tested, namely EGFP, EYFP, Citrine and PhiYFP had $\varepsilon \leq 10 \%$ and $\tau>1 \mathrm{~ms}$ in aqueous media and were thus suitable for GSDIM (Supplementary Table 1). The GSDIM image of living PtK2 cells expressing Citrine fused to the mictotubule-associated protein Map2, resulting in the labeling of the microtubule cytoskeleton, illustrates a marked resolution gain (Fig. 2f,g), yet the resolution $(\sim 40 \mathrm{~nm})$ and the contrast were still somewhat lower than in immunostained cells (Fig. 2a-e). This stems from the fact that switching to the dark state is usually faster for the fluorescent proteins than for the organic dyes, restricting the number $m$ of photons in a bunch (Supplementary Methods). Furthermore, the average offtime of a Citrine molecule in this molecular construct is rather long (>1 s; Supplementary Table 1), which limits the number of on-off cycles before irreversible bleaching and reduces the contrast of the reconstructed GSDIM image. In comparison, Rh6G molecules return faster, are more photostable and return more often. Nevertheless, the stronger photobleaching of Citrine seems not to be of fundamental nature because the lifetime of the dark state of these proteins in a pure aqueous environment is much shorter $(50 \mathrm{~ms})$, implying that Citrine is generally more photostable than in the example above. Although the substantial variation in lifetime can be attributed to changes in the $\mathrm{pH}$ or in the concentration of molecular oxygen (Supplementary Table 1), this example highlights the relevance of the molecular environment in a particular application of GSDIM.

Fluorescence depletion, instead of activation, provides a conventional first image, giving an overview of the sample and an indication when to stop the stochastic picture assembly. As we allowed less than one singlet-state molecule to be present in the diffraction area, the allowable total number of usable fluorophores within this area was $1 / \mathcal{E} \approx \Phi_{\text {isc }} \tau / \tau_{\mathrm{fl}}$, which is a function of the dye and the environment. A remedy for densely labeled samples is to bleach some of the fluorophores before imaging (Fig. 2) or 'shelve' them in a very long-lived dark state. Likewise, the return rate $1 / \tau$ of the few, remaining fluorophores may be too low at later camera frames, slowing down the image acquisition. In this case, the return can then be accelerated by additional light depopulating the dark state $\left(T_{1}\right.$ or $\left.D\right)$ via dark-state absorption ${ }^{17}$ (Supplementary Fig. 1 online). We applied this procedure by also illuminating with 

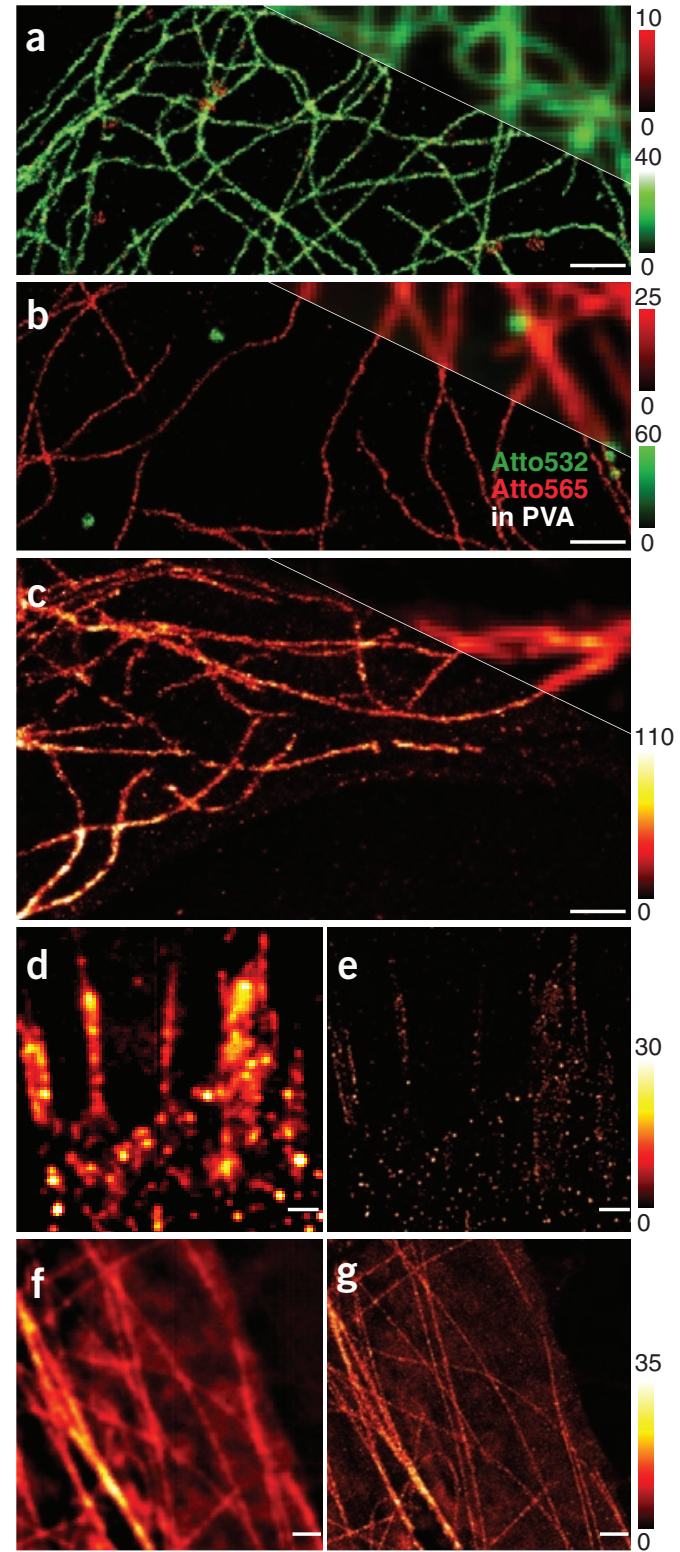

Figure 2 | Subdiffraction resolution images recorded by GSDIM. (a,b) GSDIM images of immunostained (Att0532, green or Atto565, red) microtubules and peroxysomes of PtK2-cells embedded in PVA. Epifluorescence images are shown in the upper right corners. In $\mathbf{b}$ the fluorophore labels are reverted with respect to those in a. (c) Immunostained (Rh6G) microtubules of PtK2-cells in aqueous buffer. (d,e) Diffraction-limited (d) and GDSIM (e) recordings of immunostained (Atto532) integrin- $\beta$-3 clusters of human glioma cells in a cell medium. $(\mathbf{f}, \mathbf{g})$ Epifluorescence $(\mathbf{f})$ and GSDIM $(\mathbf{g})$ images of the microtubule cytoskeleton of living PtK2 cells labeled with Citrine-Map2. Scale bars, $1 \mu \mathrm{m}$. Color bars indicate the number of events localized per spot. Number of camera frames: 72,000 (a), 82,000 (b), 61,000 (c) and $31,000(\mathbf{d}-\mathbf{g})$; the epifluorescence images were obtained by adding the total signal of all the frames. Camera frame rate: $100 \mathrm{~Hz}(\mathbf{a}, \mathbf{b}), 200 \mathrm{~Hz}$ $(\mathbf{c}-\mathbf{g})$. Laser intensity and wavelength: $10 \mathrm{~kW} / \mathrm{cm}^{2}(\mathbf{a}), 20 \mathrm{~kW} / \mathrm{cm}^{2}(\mathbf{b}), 115$ $\mathrm{kW} / \mathrm{cm}^{2}(\mathbf{c}-\mathbf{e}), 2.5 \mathrm{~kW} / \mathrm{cm}^{2}(\mathbf{f}, \mathbf{g})$ at $532 \mathrm{~nm}(\mathbf{a}-\mathbf{e})$ and $488 \mathrm{~nm}(\mathbf{f}, \mathbf{g})$. Total number of events: 413,668 (green) and 39,694 (red) (a), 29,819 (green) and 176,443 (red) (b), 870,699 (c), 130,117 (d,e) and 738,395 (f,g). $375-\mathrm{nm}$ light in the recordings shown in Figure 2 starting at image frame number $\sim 20,000$ in Figure 2a, and image frame number $\sim 10,000$ in Figure $2 \mathbf{b}$,e; we used no additional light in the recordings shown in Figure 2c,g. Note that this illumination is optional and applied to regular fluorophores. Unlike GSD microscopy $y^{2,3}$, GSDIM requires a fluorophore to recover to $S_{0}$ only once. By stochastically recording the position of individual molecules, GSDIM substantially differs from GSD microscopy, which defines the position of molecular detection with intensity zeros. Yet both concepts rely on the same molecular mechanism, a fact that highlights switching between a dark and a bright state as the actual element enabling super-resolution imaging. As switching is not restricted to fluorescence, other contrast modes, such as changes in the scattering spectrum of (paired) collodial particles, are poised to be implemented as well ${ }^{6}$.

In any case, the intensity-zero, ensemble-based approach and the stochastic single-molecule-based approach remain complementary modalities of determining the position of nearby molecules. The latter modality has the advantage of requiring fewer switching cycles, which also accounts for the fact that GSDIM currently appears to be more readily applicable than GSD microscopy. Moreover, GSDIM is strikingly simple: continual epifluorescence recording with a freely operating camera allows the computational construction of nanoscale images with multiple standard dyes and fluorescent proteins.

Note: Supplementary information is available on the Nature Methods website.

\section{ACKNOWLEDGMENTS}

We thank A. Egner and A. Schönle for support with the instrumentation and data evaluation software and for valuable discussions, R.Y. Tsien (University of California, San Diego) for providing the plasmid coding for fluorescent proteins, V.N. Belov (Max Planck Institute for Biophysical Chemistry, Göttingen) for supplying the rhodamine dyes Rh-sart3b and Rh-sart3f, R. Schmitz-Salue and S. Löbermann for excellent technical assistance, S. Sahl for help with the experiments, and B. Rankin and J. Jethwa for carefully reading the manuscript.

\section{Published online at http://www.nature.com/naturemethods/} Reprints and permissions information is available online at http://npg.nature.com/reprintsandpermissions/

1. Hell, S.W. \& Wichmann, J. Opt. Lett. 19, 780-782 (1994).

2. Hell, S.W. \& Kroug, M. Appl. Phys. B 60, 495-497 (1995).

3. Bretschneider, S., Eggeling, C. \& Hell, S.W. Phys. Rev. Lett. 98, 218103 (2007).

4. Heintzmann, R., Jovin, T.M. \& Cremer, C. J. Opt. Soc. Am. A 19, 1599-1609 (2002).

5. Gustafsson, M.G.L. Proc. Natl. Acad. Sci. USA 102, 13081-13086 (2005).

6. Hell, S.W., Jakobs, S. \& Kastrup, L. Appl. Phys. A 77, 859-860 (2003).

7. Hell, S.W. Science 316, 1153-1158 (2007).

8. Betzig, E. et al. Science 313, 1642-1645 (2006).

9. Hess, S.T., Girirajan, T.P.K. \& Mason, M.D. Biophys. J. 91, 4258-4272 (2006).

10. Rust, M.J., Bates, M. \& Zhuang, X. Nat. Methods 3, 793-796 (2006).

11. Heisenberg, W. The Physical Principles of the Quantum Theory. (University of Chicago Press, Chicago, 1930).

12. Fölling, J. et al. Angew. Chem. Int. Ed. 46, 6266-6270 (2007).

13. Bock, H. et al. Appl. Phys. B 88, 161-165 (2007).

14. Heilemann, M. et al. Angew. Chem. 47, 6172-6176 (2008).

15. Zondervan, R., Kulzer, F., Orlinskii, S.B. \& Orrit, M. J. Phys. Chem. A 107, 6770-6776 (2003)

16. Bossi, M. et al. Nano Lett. 8, 2463-2468 (2008).

17. Dickson, R.M., Cubitt, A.B., Tsien, R.Y. \& Moerner, W.E. Nature 388, 355-358 (1997). 